OnÉSIMO Hernández-LERma (México)

JEAN B. LASSERRE (Toulouse)

\title{
ON THE PROBABILISTIC MULTICHAIN POISSON EQUATION
}

Abstract. This paper introduces necessary and/or sufficient conditions for the existence of solutions $(g, h)$ to the probabilistic multichain Poisson equation
(a) $g=P g$ and
(b) $g+h-P h=f$,

with a given charge $f$, where $P$ is a Markov kernel (or transition probability function) on a general measurable space. The existence conditions are derived via three different approaches, using (1) canonical pairs, (2) Cesàro averages, and (3) resolvents.

1. Introduction. Let $(X, \mathcal{B})$ be a measurable space, and $P(x, B)$ a Markov kernel (or transition probability function) on $(X, \mathcal{B})$, that is, $P(x, \cdot)$ is a probability measure on $\mathcal{B}$ for every fixed $x \in X$, and $P(\cdot, B)$ is a measurable function on $X$ for every fixed $B \in \mathcal{B}$. For a real-valued measurable function $h$ on $X$, we denote by $P h$ the function

$$
P h(x):=\int_{X} P(x, d y) h(y), \quad x \in X,
$$

whenever the integral is well defined. The Poisson equation (P.E.) for $P$ is

$$
\text { (a) } g=P g \quad \text { and } \quad \text { (b) } g+h-P h=f \text {, }
$$

where $f: X \rightarrow \mathbb{R}$ is a given measurable function, called a charge. If (1.1) holds, then the pair $(g, h)$ is said to be a solution to the P.E. with charge $f$.

2000 Mathematics Subject Classification: 60J05, 60J45.

Key words and phrases: discrete-time Markov processes, Poisson's equation, mean ergodic theorem, canonical pairs, Cesàro averages, resolvent, potentials.

This research was partially supported by the CNRS (France)-CONACYT (México) and the ECOS-Nord Scientific Cooperation Programs. The work of the first author (OHL) was also partially supported by CONACYT Grant 32299-E. 
If it is known that $P$ admits a unique invariant probability measure, then (1.1) is called the unichain P.E.; otherwise, (1.1) is called the multichain (or general) P.E. The problem we are concerned with in this paper is to obtain necessary and/or sufficient conditions for the existence of solutions to the multichain P.E.

The P.E. for discrete (or continuous) time Markov processes occurs in many areas of applied and theoretical probability, including potential theory, stochastic approximation and stochastic control [10, 11-14, 17, 18, 25, 26, $28-31,36,41,42]$. In particular, in the analysis of average (or ergodic) cost problems for Markov control processes, (1.1) is a special case of the so-called average-cost optimality equation, also known as Bellman's equation, and the existence of solutions to this equation is, among other things, required to analyze the policy iteration (or Howard's) algorithm [1, 6, 13, 14, 30].

A relevant question then is: How can we ensure the existence of solutions to the P.E.? There are two cases in which the answer to this question is well known. One is when the state space $X$ is a countable set (see Remark 2.6 below), and the other is the above-mentioned unichain case, in which the function $g$ in (1.1) turns out to be a constant (for a special situation, see Remark 3.6). In these two cases, a key probabilistic fact used to obtain a solution to (1.1) is that the "ergodic decomposition" of $X$ in "recurrent" and "transient" parts is well understood. These concepts, however, do not have a unique meaning in the multichain situation with a general space $X$; namely, one can have several types of "ergodic decompositions" (Doeblin's, Harris', Hopf's, ... ), and even then to obtain (1.1) one further needs suitable topological assumptions on $X$ and/or probabilistic hypotheses (for instance, the Feller condition) on the transition probability $P$. A consequence of this is that if one wishes to study the multichain P.E. with general $X$ and $P$, a "probabilistic" approach does not look very promising. We have to try other methods. Here we try several alternative, basically functional-analytic approaches.

In this paper we follow three (related) approaches to study (1.1). In Section 2 we show that the existence of a solution to (1.1) is equivalent to the existence of a canonical pair (Definition 2.1). The latter shows in particular the close relation between (1.1) and the existence of limits (as $n \rightarrow \infty)$ of Cesàro averages

$$
A_{n} f:=n^{-1} \sum_{k=0}^{n-1} P^{k} f .
$$

Hence, in Section 3 we turn our attention to the Mean Ergodic Theorem of Yosida and Kakutani [40], which gives a precise description of the set of functions $f$ for which $A_{n} f$ converges (see Theorem 3.2), and allows us to 
fully determine (Theorem 3.7) the set of solutions to (1.1). An alternative description of this set is obtained in Section 4 (Theorem 4.3) using the well known relation between the limiting behaviour of the Cesàro averages $A_{n} f$ and the limit as $\alpha \uparrow 1$ of $(1-\alpha) R_{\alpha} f$, where

$$
R_{\alpha} f:=\sum_{k=0}^{\infty} \alpha^{k} P^{k} f \quad \text { with } 0<\alpha<1,
$$

is the resolvent (or $\alpha$-potential) of $P$. The results in Sections 3 and 4 are strongly influenced by the work of previous authors on the existence of solutions to linear equations in Banach spaces [2, 23, 33, 35]. (In a somewhat related context but using a technically different approach, via a generalized Farkas theorem, existence results are also presented in $[12,16]$.) Finally, the paper concludes in Section 5 with a brief description of some open problems.

2. Canonical pairs. Let $(\mathcal{X},\|\cdot\|)$ be a normed vector space of realvalued bounded measurable functions on $(X, \mathcal{B})$. [In most applications of Markov processes, $\mathcal{X}$ is in fact a Banach space, for instance, $\mathcal{X}=L_{p}(X, \mathcal{B}, \mu)$ for some $\sigma$-finite measure $\mu$ and $1 \leq p \leq \infty$, or the space $\mathcal{X}=\mathbb{B}(X)$ of bounded measurable functions on $(X, \mathcal{B})$ with the supremum norm.] Unless otherwise stated, convergence in $\mathcal{X}$ is always in the strong (norm) topology: " $f_{n} \rightarrow f$ strongly" means $\left\|f_{n}-f\right\| \rightarrow 0$. In this case, we write $f=s$-lim $f_{n}$. For operators on $\mathcal{X}$ strong convergence $T:=s-\lim T_{n}$ means $T f=s-\lim T_{n} f$ for all $f \in \mathcal{X}$.

We shall assume that the transition probability function $P$ defines a linear operator on $\mathcal{X}$ into itself given by

$$
(P f)(x):=\int_{X} P(x, d y) f(y), \quad \forall f \in \mathcal{X}, x \in X .
$$

As usual, $P^{n}$ denotes the $n$-step transition function, which is given recursively by

$$
P^{n}(x, B)=\int_{X} P^{n-1}(x, d y) P(y, B), \quad n=1,2, \ldots,
$$

where $P^{0}(x, \cdot)$ is the Dirac measure at $x$; we also write $P^{0}:=I$, the identity operator. For $n=1,2, \ldots$, let

$$
S_{n}:=I+P+\ldots+P^{n-1} \text { and } A_{n}:=\frac{1}{n} S_{n}
$$

The $A_{n}$ are sometimes called the Cesàro (or ergodic) averages of $P$.

The following definition is an adaptation of the concept of canonical triplet introduced by Yushkevich [41] (see also [6] or [13]) for Markov control processes. 
Definition 2.1. Let $f$ be a given function in $\mathcal{X}$. A pair $(g, h)$ of functions $g$ and $h$ in $\mathcal{X}$ is said to be an $f$-canonical pair if

$$
S_{n} f+P^{n} h=n g+h \quad \forall n=1,2, \ldots
$$

It turns out that (2.2) is equivalent to the multichain P.E. (1.1) in the following sense.

Theorem 2.2. $(g, h)$ is an $f$-canonical pair if and only if $(g, h)$ is a solution to the multichain P.E. with charge $f$.

Proof. " $\Rightarrow$ " Let $(g, h)$ be an $f$-canonical pair. Then, with $n=1,(2.2)$ yields (1.1)(b). Now, to obtain (1.1)(a), apply $P$ to both sides of (1.1)(b) to get

$$
P^{2} h=P g+P h-P f,
$$

and, on the other hand, note that (2.2) with $n=2$ yields

$$
P^{2} h=2 g+h-f-P f .
$$

The last two equations give (1.1)(a) since they imply

$$
P g-g=g+h-P h-f=0,
$$

where the latter equality comes from $(1.1)(\mathrm{b})$.

" $\Leftarrow$ " Conversely, suppose that $(g, h)$ satisfies (1.1). Then $g=P g$ implies $g=P^{k} g$ for all $k=0,1, \ldots$ and, therefore,

$$
n g=\sum_{k=0}^{n-1} P^{k} g=S_{n} g \quad \forall n=1,2, \ldots
$$

Now write (1.1)(b) as $h=(f-g)+P h$ and iterate to obtain

$$
h=S_{n}(f-g)+P^{n} h=S_{n} f-n g+P^{n} h \quad[\text { by }(2.3)],
$$

which is the same as (2.2).

Although Theorem 2.2 is quite straightforward, it has important consequences. In particular, we will derive from it additional necessary and/or sufficient conditions for the existence of solutions to the multichain P.E. Recall that the norm of an operator $T$ on $\mathcal{X}$ is defined as

$$
\|T\|:=\sup \{\|T f\| \mid f \in \mathcal{X},\|f\| \leq 1\},
$$

and that $T$ is said to be power-bounded if there is a constant $M \geq 0$ such that $\left\|T^{n}\right\| \leq M$ for all $n=0,1, \ldots$

Corollary 2.3. Let $(g, h)$ be an $f$-canonical pair. Then:

(a) $g=s-\lim _{n} A_{n} g$.

(b) If $P^{n} h / n \rightarrow 0$ (pointwise or strongly), then $\lim A_{n} f=\lim A_{n} g=g$ (pointwise or strongly, respectively).

(c) If $P$ is power-bounded, then $\sup _{n}\left\|S_{n}(f-g)\right\|<\infty$. 
Proof. Part (a) follows from (2.3). Moreover, from (2.3) and (2.2),

$$
S_{n}(f-g)=h-P^{n} h .
$$

This yields (b) [see (a)], and also (c) since $\left\|S_{n}(f-g)\right\| \leq(1+M)\|h\|$, where $M$ is such that $\left\|P^{n}\right\| \leq M$ for all $n=0,1, \ldots$

REMARK 2.4. (a) Observe that if (1.1)(b) holds, so that $f-g=(I-P) h$, we can also obtain (2.4) from the general expression:

$$
S_{n}(I-P)=\sum_{k=0}^{n-1} P^{k}(I-P)=I-P^{n} \quad \forall n=1,2, \ldots
$$

(b) The hypotheses in parts (b) and (c) of Corollary 2.3 obviously hold if $P$ is a contraction operator, i.e., $\|P\| \leq 1$. This is the case if, for instance, $\mathcal{X}=\mathbb{B}(X)$ is the Banach space of bounded measurable functions on $(X, \mathcal{B})$ with the sup norm, or if $\mathcal{X}=L_{p}(X, \mathcal{B}, \mu)$ with $1 \leq p \leq \infty$ and $\mu$ a $P$ invariant probability measure, i.e., $\mu$ is a (not necessarily unique) probability measure such that

$$
\mu(B)=\int_{X} \mu(d x) P(x, B) \quad \forall B \in \mathcal{B} .
$$

The following theorem gives another characterization of a solution to $(1.1)$.

Theorem 2.5. Let $f, g$ and $h$ be functions in $\mathcal{X}$, and suppose that:

(a) $P$ is bounded (i.e., $\|P\| \leq M$ for some constant $M$ ), and

(b) $P^{n} / n \rightarrow 0$ strongly.

Then the following two assertions are equivalent.

(i) $(g, h)$ is the unique solution of the P.E. (1.1) for which

$$
s-\lim A_{n} h=0 .
$$

(ii) $g=s-\lim A_{n} g=s-\lim A_{n} f$, and

$$
h=s-\lim \frac{1}{N} \sum_{n=1}^{N} \sum_{k=0}^{n-1} P^{k}(f-g)=s-\lim \frac{1}{N} \sum_{n=1}^{N} S_{n}(f-g) .
$$

Proof. (i) $\Rightarrow$ (ii). If (i) holds, then the first condition in (ii) follows from Corollary 2.3(b). On the other hand, by (2.4),

$$
h=\frac{1}{N} \sum_{n=1}^{N} S_{n}(f-g)-\frac{1}{N} \sum_{n=1}^{N} P^{n} h \quad \forall N=1,2, \ldots
$$

Hence, (2.8) follows from (2.9) and (2.7). 
(ii) $\Rightarrow$ (i). If $g=s$-lim $A_{n} g=s$-lim $A_{n} f$, then (1.1)(a) holds [since, by assumption (a), we can interchange $P$ and $s$-lim], and also

$$
s-\lim A_{n}(f-g)=0 .
$$

To prove (1.1)(b) first note that, by (2.5),

$$
(I-P) \sum_{k=0}^{n-1} P^{k}(f-g)=\left(I-P^{n}\right)(f-g) .
$$

Therefore, applying $I-P$ to both sides of (2.8) and using assumption (a) again, we get

$$
\begin{aligned}
(I-P) h & =s-\lim \frac{1}{N} \sum_{n=1}^{N}(I-P) \sum_{k=0}^{n-1} P^{k}(f-g) \\
& =(f-g)-s-\lim \frac{1}{N} \sum_{n=1}^{N} P^{n}(f-g) \\
& =f-g \quad[\text { by }(2.10)],
\end{aligned}
$$

i.e., (1.1)(b) holds. Hence, the pair $(g, h)$ is a solution to (1.1); it only remains to show that it is unique. Before doing this, let us note that (2.8) and (2.9) together imply $(2.7)$. Now let $\left(g_{1}, h_{1}\right)$ and $\left(g_{2}, h_{2}\right)$ be two $f$-canonical pairs satisfying the conditions in (ii). Then

$$
g_{1}=s-\lim A_{n} f=g_{2}, \quad \text { i.e., } \quad g_{1}=g_{2}=: g .
$$

Furthermore, since $(I-P) h_{i}=f-g$ for $i=1,2$, the function $u=h_{1}-h_{2}$ satisfies $(I-P) u=0$, and, therefore, $u=P^{k} u$ for all $k=0,1, \ldots$, which implies

$$
u=s-\lim A_{n} u=s-\lim A_{n} h_{1}-s-\lim A_{n} h_{2}=0 \quad[\text { by }(2.7)],
$$

i.e., $h_{1}=h_{2}$.

In the following section we show that the results in Theorem 2.5, as well as those mentioned in Remark 2.6, below, are valid in a more general context.

REMARK 2.6. If the state space $X$ is a finite set, in which case the transition probability $P$ is a square matrix, it is well known $[1,6,18,25,30]$ that the limiting matrix

$$
\Pi:=\lim _{n} A_{n}=\lim _{n} n^{-1} \sum_{k=0}^{n-1} P^{k} \text { (componentwise) }
$$

exists, and that $I-P+\Pi$ is nonsingular; its inverse

$$
Z:=(I-P+\Pi)^{-1}
$$


is called the fundamental matrix associated with $P$. Moreover, the matrix

satisfies

$$
H:=\lim _{N \rightarrow \infty} \frac{1}{N} \sum_{n=1}^{N} \sum_{k=0}^{n-1}(P-\Pi)^{k}(I-\Pi)
$$

$$
H=(I-P+\Pi)^{-1}(I-\Pi)=Z(I-\Pi)
$$

and is called the deviation matrix associated with $P$ (or the Drazin inverse of $I-P) ; P-\Pi$ is sometimes called the approach matrix [36]. The above facts are also true if $X$ is a countable set. What we wish to remark is that the solution pair $(g, h)$ in Theorem $2.5(\mathrm{i}),(\mathrm{ii})$ is precisely

$$
g=\Pi f \quad \text { and } \quad h=H f \text {. }
$$

In Theorem 3.7 we will show that (2.16) and (2.17) hold in a much more general setting.

REMARK 2.7. The choice of the underlying space $\mathcal{X}$ is important. For instance, consider the countable set $X=\{1,2, \ldots\}$ with the discrete topology, and let $\mathcal{X}$ be the Banach space of bounded functions on $X$ with the supremum norm $\|u\|:=\sup _{x}|u(x)|$. Further, let $\{q(x)\}, x \in X$, be a probability distribution on $X$, that is, $q(x) \geq 0$ for all $x$ and $\sum_{x} q(x)=1$, which is assumed to have a finite "mean value":

$$
\bar{q}:=\sum_{x} x q(x)<\infty
$$

and let $P(x, y) \equiv P(x,\{y\})$ be the Markov kernel given by

$$
P(x, x-1):=1 \quad \forall x \geq 2, \quad \text { and } \quad P(1, y):=q(y) \quad \forall y \geq 1 .
$$

Finally, consider the Poisson equation (1.1) with charge $f \in \mathcal{X}$ defined by

$$
f(1):=1-\bar{q}, \quad \text { and } \quad f(x):=1 \quad \forall x \geq 2 .
$$

Then one can easily check that (1.1) has a solution $(h, g)$ with $g(\cdot) \equiv 0$ and

$$
h(x)=f(1)+x-1 \quad \forall x \in X .
$$

In fact, except for an additive constant, any solution $h$ to (1.1) is of the form (2.18), which is not a bounded function. In other words, the charge $f$ is in $\mathcal{X}$ and the P.E. is "solvable", but the solution is not in $\mathcal{X}$. This kind of situation can often be remedied by suitably enlarging the space $\mathcal{X}$. For example, consider the weighted norm

$$
\|u\|_{w}:=\|u / w\|=\sup _{x}|u(x)| w(x)^{-1},
$$

where $w(x)=x$ for all $x \in X$, and let $\mathcal{X}_{w}$ be the Banach space of functions $u$ on $X$ with finite $w$-norm, i.e., $\|u\|_{w}<\infty$. It is clear that $\mathcal{X}_{w}$ contains $\mathcal{X}$ (in fact, since $w \geq 1$, we have $\|u\|_{w} \leq\|u\|<\infty$ if $u$ is bounded) and, moreover, the function $h$ in (2.18) belongs to $\mathcal{X}_{w}$. That is, the P.E. does not have a 
solution in $\mathcal{X}$, but it does in $\mathcal{X}_{w}$. Moreover, it is straightforward to check that $P$ is still a bounded linear operator on $\mathcal{X}_{w}$. Under some additional assumption on the distribution $q$ (for instance, if $q$ has a finite "moment generating function"), one can show that $P$ is in fact power-bounded.

3. The Cesàro-averages approach. It follows from the results in Section 2 that the existence of solutions to the multichain P.E. (or, equivalently, the existence of canonical pairs) is closely connected with the limiting behaviour of the Cesàro averages $A_{n}:=n^{-1} S_{n}$. In this section we obtain necessary and/or sufficient conditions for the existence of such solutions by identifying the limits of $A_{n}$. To do this we shall use the Mean Ergodic Theorem of Yosida and Kakutani [40] (see also [39] or [5]), which requires the following assumption.

Assumption 3.1. $\mathcal{X}$ is a Banach space and $P$ maps $\mathcal{X}$ into itself. Moreover,

(a) $P^{n} / n \rightarrow 0$ strongly, and

(b) $\sup _{n}\left\|A_{n}\right\|<\infty$.

Note that (a) and (b) trivially hold if $P$ is power-bounded, in particular, if $P$ is a contraction [see Remark 2.4(b)].

Now let $A(P)$ be the set of functions whose Cesàro averages converge, i.e.,

$$
A(P):=\left\{f \in \mathcal{X} \mid A_{n} f \text { converges strongly as } n \rightarrow \infty\right\} .
$$

The set $A(P)$ is non-empty [it contains (at least) the constant functions] and the following Mean Ergodic Theorem (for a proof see the above-mentioned references) provides a description of it. We use the notation Ker $:=$ kernel (or null) space and Ran $:=$ range.

Theorem 3.2. Suppose that Assumption 3.1 holds. Then $A(P)$ is the closed linear manifold given by

$$
A(P)=\operatorname{Ker}(I-P) \oplus \overline{\operatorname{Ran}(I-P)} .
$$

Furthermore, the operator $\Pi$ that maps $f \mapsto \Pi f:=s-\lim A_{n} f$ is a projection on $A(P)$ with

$$
\operatorname{Ran}(\Pi)=\operatorname{Ker}(I-P), \quad \operatorname{Ker}(\Pi)=\overline{\operatorname{Ran}(I-P)},
$$

and satisfies

$$
\Pi P=P \Pi=\Pi^{2}=\Pi .
$$

If , in addition, $\mathcal{X}$ is reflexive then $P$ is mean ergodic, i.e., $A(P)=\mathcal{X}$.

Remark 3.3. Concerning the last statement in Theorem 3.2, the condition that $\mathcal{X}$ be reflexive is sufficient but not necessary for $P$ to be mean ergodic. For instance, suppose that $\mu$ is a P-invariant probability measure 
[see Remark 2.4(b)] and let $\mathcal{X}=L_{1}(X, \mathcal{B}, \mu)$. Then $\mathcal{X}$ is not reflexive but it is well known that $A(P)=\mathcal{X}$. Moreover, $\Pi f$ is given by a conditional expectation $[8,31]$, say

$$
\Pi f=E(f \mid \mathcal{I}) \quad \forall f \in \mathcal{X}=L_{1}(X, \mathcal{B}, \mu)
$$

(where $\mathcal{I}$ is the $\sigma$-algebra of "invariant" sets), and, on the other hand, if $X$ is a locally compact separable metric space and $\mathcal{B}$ is the corresponding Borel $\sigma$-algebra, then (with a.a. $=$ almost all)

$$
\Pi f(x)=\int_{X} \Pi(x, d y) f(y) \quad \text { for } \mu \text {-a.a. } x \in X,
$$

where $\Pi(x, B)$ is a transition probability function. (See [15].)

We shall now derive necessary conditions for the existence of solutions to (1.1); sufficient conditions are considered in the second half of this section.

Let $(g, h)$ be a solution of the multichain P.E. with charge $f$, and suppose that Assumption 3.1 holds. Then, by (3.1) and (1.1), $g$ and $f$ are both in $A(P)$, and in fact, by Corollary $2.3(\mathrm{a}),(\mathrm{b})$,

$$
g=\Pi f \text {. }
$$

Hence, in particular, we may rewrite (1.1)(b) as

$$
(I-P) h=(I-\Pi) f .
$$

On the other hand, by (3.4), $g$ is necessarily unique but this need not be the case for $h$ because $\left(g, h+\Pi h^{\prime}\right)$ is also a solution of the multichain P.E. for any $h^{\prime}$ in $A(P)$; indeed, by (3.3),

$$
(I-P)\left(h+\Pi h^{\prime}\right)=(I-P) h=(I-\Pi) f .
$$

For $h$ to be unique it suffices to add the constraint

$$
\Pi h=0 .
$$

In other words, as in the last part of the proof of Theorem 2.5, we have:

Proposition 3.4. If $\left(g, h_{1}\right)$ and $\left(g, h_{2}\right)$ are two solutions of the multichain P.E. and $h_{1}, h_{2}$ satisfy (3.6), then $h_{1}=h_{2}$.

Proof. From (3.5), we have $(I-P)\left(h_{1}-h_{2}\right)=0$, i.e., $u:=h_{1}-h_{2}$ is in $\operatorname{Ker}(I-P)=\operatorname{Ran}(\Pi)$ [by (3.2)]. This implies $u=\Pi u$, so that, by (3.6), $u=h_{1}-h_{2}=0$.

Finally, we shall use (2.5) to re-state Corollary 2.3 in the context of this section. Actually, the following proposition almost amounts to a trivial remark but it is important because it gives an idea of the rate of convergence of $A_{n} f$ to $\Pi f$. 
Proposition 3.5. Suppose that Assumption 3.1 holds. If $f$ and $h$ satisfy (3.5), with $f$ in $A(P)$, then

$$
A_{n} f-\Pi f=\frac{1}{n}\left(I-P^{n}\right) h \rightarrow 0 \quad \text { strongly. }
$$

If , in addition, $P$ is power-bounded then

$$
\left\|A_{n} f-\Pi f\right\| \leq M\|h\| / n \text {. }
$$

for some constant $M$.

Proof. From (3.5) and (2.5),

$$
S_{n}(I-\Pi) f=S_{n}(I-P) h=\left(I-P^{n}\right) h .
$$

Since $S_{n}(I-\Pi)=S_{n}-n \Pi$, we get (3.7), hence also (3.8).

REMARK 3.6. The convergence in (3.8) can be greatly improved by imposing suitable assumptions on the transition probability function $P$. In particular, there is a large variety of conditions ensuring a geometric rate of convergence, that is, there exists a constant $0<\beta<1$ such that

$$
\left\|P^{n} f-\mu(f)\right\| \leq c \beta^{n} \quad \forall f \in \mathcal{X} \quad \text { and } \quad n=0,1, \ldots,
$$

where $\mu(f):=\int f d \mu=\Pi f$ for some $P$-invariant probability measure $\mu$, and $c$ is a constant (that may depend on $f$ ). See $[4,10,11,17,28]$.

Note that if (3.9) holds, then the operator $H_{0}$ introduced below is defined for all $f \in \mathcal{X}$.

To state sufficient conditions for the existence of solutions to the multichain P.E., consider two operators $H_{0}$ and $H$ defined as

$$
H_{0} f:=s-\lim _{n \rightarrow \infty} \sum_{k=0}^{n-1}\left(P^{k}-\Pi\right) f=\sum_{k=0}^{\infty}\left(P^{k}-\Pi\right) f,
$$

and

$$
H f:=s-\lim _{N \rightarrow \infty} \frac{1}{N} \sum_{n=1}^{N} \sum_{k=0}^{n-1}\left(P^{k}-\Pi\right) f .
$$

The domain of $H$ is $\operatorname{Dom}(H):=\{f \in A(P) \mid$ the limit in (3.10) exists $\}$, and similarly for $H_{0}$. If a sequence $\left\{h_{n}\right\}$ in $\mathcal{X}$ converges strongly to $h$, then so does the sequence of averages $n^{-1} \sum_{k=0}^{n-1} h_{k}$. Thus, taking

$$
h_{n}:=\sum_{k=0}^{n-1}\left(P^{k}-\Pi\right) f
$$

we see that $H$ is an extension of $H_{0}$, that is, $\operatorname{Dom}\left(H_{0}\right) \subset \operatorname{Dom}(H)$ and

$$
H f=H_{0} f \quad \forall f \in \operatorname{Dom}\left(H_{0}\right) .
$$

In fact, these remarks were intended mainly to illustrate the relation between (3.9) and $H_{0}$, whence between (3.9) and $H$. But what we are really 
interested in is the following result, which in particular gives the precise domain and range of $H$. [Compare Theorem 3.7 and Remark 2.6, noting that $(P-\Pi)^{k}(I-\Pi)=P^{k}-\Pi$ for all $k=0,1, \ldots$, by $\left.(3.3).\right]$

TheOREM 3.7. Under Assumption 3.1 we have:

(a) $f$ is in $\operatorname{Dom}(H)$ if and only if the pair $(g, h)$ given by $g=\Pi f$ and

$$
h:=H f=s-\lim _{N \rightarrow \infty} N^{-1} \sum_{n=1}^{N} \sum_{k=0}^{n-1}\left(P^{k}-\Pi\right) f
$$

is the unique solution of (3.4)-(3.6).

(b) $\operatorname{Dom}(H)=\operatorname{Ran}(\Pi) \oplus(I-P) \operatorname{Ker}(\Pi)[=\operatorname{Ker}(I-P) \oplus(I-P) \overline{\operatorname{Ran}(I-P)}$, by (3.2)].

(c) $\operatorname{Ran}(H)=\operatorname{Ker}(\Pi)[=\overline{\operatorname{Ran}(I-P)}$, by $(3.2)]$.

(d) The restriction of $H$ to $\operatorname{Ran}(H)=\operatorname{Ker}(\Pi)$, call it $Z$, is the inverse of $I-P+\Pi$, i.e.,

$$
Z f=(I-P+\Pi)^{-1} f \quad \forall f \in \operatorname{Ran}(H)[=\operatorname{Ker}(\Pi), b y(\mathrm{c})] ;
$$

hence, the function $h$ in (3.11) can be written as

$$
h=H f=Z(I-\Pi) f \quad \forall f \in \operatorname{Dom}(H) .
$$

Proof. (a) Suppose that $f$ is in $\operatorname{Dom}(H)$, and let $g:=\Pi f$ and $h:=H f$. Then observing that [by (3.3)]

$$
P^{k}(I-\Pi) f=\left(P^{k}-\Pi\right) f \quad \forall k=0,1, \ldots,
$$

we see that the function $h=H f$ in (3.11) is the same as the function $h$ in $(2.8)$ with $g=\Pi f$. Hence the implication " $\Rightarrow$ " in (a) follows from the implication "(ii) $\Rightarrow$ (i)" in Theorem 2.5. Similarly, the converse follows from "(i) $\Rightarrow$ (ii)" in Theorem 2.5 .

(b) Let $f$ be in $\operatorname{Dom}(H)$ and let $g:=\Pi f$ and $h:=H f$. Then from part (a), (3.5) and (3.6) yield

$$
f=\Pi f+(I-P) h \quad \text { with } h \in \operatorname{Ker}(\Pi) ;
$$

hence

$$
f \in \operatorname{Ran}(\Pi) \oplus(I-P) \operatorname{Ker}(\Pi) .
$$

Now suppose that $f$ satisfies (3.14). Then there are functions $f_{1}$ in $A(P)$ and $f_{2}$ in $\operatorname{Ker}(\Pi)$ such that $f=\Pi f_{1}+(I-P) f_{2}$. Obviously [by $\left.(3.3)\right], \Pi f_{1}$ is in $\operatorname{Dom}(H)$ and $H \Pi f_{1}=0$. Moreover, since [by (3.3) again]

$$
\left(P^{k}-\Pi\right)(I-P)=P^{k}-P^{k+1},
$$

$H(I-P) f_{2}=f_{2}$. Summarizing, if $f$ satisfies (3.14), then $f$ is in $\operatorname{Dom}(H)$ and $H f=f_{2}$.

(c) Suppose $h=H f$ is in $\operatorname{Ran}(H)$. Then since $\Pi$ is bounded (Theorem $3.2)$, we can interchange $\Pi$ and $s$-lim in (3.10), which combined with (3.3) 
yields

$$
\Pi h=\Pi H f=0, \quad \text { i.e., } \quad h \in \operatorname{Ker}(\Pi),
$$

so that $\operatorname{Ran}(H) \subset \operatorname{Ker}(\Pi)$.

Now to prove that $\operatorname{Ker}(\Pi) \subset \operatorname{Ran}(H)$, let $h$ be in $\operatorname{Ker}(\Pi)$ and let $f:=$ $(I-P) h$. Then, by $(3.3)$ and $(2.5)$,

$$
\sum_{k=0}^{n-1}\left(P^{k}-\Pi\right) f=\sum_{k=0}^{n-1}\left(P^{k}-\Pi\right)(I-P) h=\left(I-P^{n}\right) h .
$$

Thus, (3.10) yields $H f=h-\Pi h=h$, i.e., $h$ is in $\operatorname{Ran}(H)$.

(d) Suppose that $f$ is in $\operatorname{Ran}(H)=\operatorname{Ker}(\Pi)$ and let $h=H f$. Then, by (a), (3.5)-(3.6) yield $(I-P) h=(I-\Pi) f=f$ and $\Pi h=0$, so that

$$
(I-P+\Pi) h=(I-P) h+\Pi h=f,
$$

i.e., $(I-P+\Pi) H=I$ on $\operatorname{Ker}(\Pi)$. By a similar argument, $H(I-P+\Pi)=I$ on $\operatorname{Ker}(\Pi)$.

Finally, to prove (3.13), let $f$ be any function in $\operatorname{Dom}(H)$. Then part (a) yields that $h=H f$ satisfies (3.5)-(3.6), so that

$$
(I-P+\Pi) H f=(I-P) h+\Pi h=(I-\Pi) f,
$$

and (3.13) follows.

REMARK 3.8. (a) Arguing as in (3.16) we can show that

$$
H(I-P) f=(I-\Pi) f \quad \forall f \in \operatorname{Dom}(H),
$$

so that in addition to (3.13) we have

$$
H(I-P+\Pi) f=H(I-P) f=(I-\Pi) f .
$$

(b) The operator $H_{0}$ defined above is sometimes called the ergodic potential of $P$, and $H_{1}:=\sum_{k=0}^{\infty} P^{k}$ is called the potential $[29,31,36]$. In the following section we study the $\alpha$-potential (or resolvent) $R_{\alpha}$ in (4.1).

4. The Abelian approach. For every $0<\alpha<1$, let $R_{\alpha}$ be the operator defined by

$$
R_{\alpha}:=(I-\alpha P)^{-1}=\sum_{k=0}^{\infty} \alpha^{k} P^{k} .
$$

The close connection between the limits of the Cesàro averages $A_{n}$ [see (2.1)] as $n \rightarrow \infty$ and the limits of the "Abelian means" $(1-\alpha) R_{\alpha}$ as $\alpha \uparrow 1$ has been widely exploited in a variety of contexts. In this section we use that connection to study the multichain P.E. (1.1). First, to ensure that, among other things, $R_{\alpha}$ is well defined, we let $\mathcal{X}$ be as in Section 3 [i.e., $\mathcal{X}$ is a Banach space of bounded measurable functions on $(X, \mathcal{B})]$ and suppose: 
Assumption 4.1. $P$ is power-bounded, i.e., there is a constant $M$ such that $\left\|P^{n}\right\| \leq M$ for all $n=0,1, \ldots$

Assumption 4.1 obviously holds, in particular, if $P$ is a contraction [see Remark 2.4(b)]. On the other hand, note that Assumption 4.1 implies Assumption 3.1 and, therefore, all the results of Section 3 are valid. Moreover,

$$
\sup _{0<\alpha<1}\left\|(1-\alpha) R_{\alpha}\right\| \leq M(<\infty)
$$

which, since $P$ is positive $(f \geq 0 \Rightarrow P f \geq 0)$, is in fact equivalent to the condition $\sup _{n}\left\|A_{n}\right\|<\infty$ in Assumption 3.1(b) (see, for instance, [7]). In addition, a well known result $[2,32]$ yields that, under Assumption 4.1, the set $A(P)$ in (3.1) is the same as the set of all $f \in \mathcal{X}$ for which the strong limit $s-\lim _{\alpha \uparrow 1}(1-\alpha) R_{\alpha} f$ exists, and in fact coincides with $\Pi f$ :

$$
s \text { - } \lim _{n \rightarrow \infty} A_{n} f=\Pi f=s \text { - } \lim _{\alpha \uparrow 1}(1-\alpha) R_{\alpha} f \quad \forall f \in A(P) .
$$

(See Remark 4.4 below.)

We will next extend to our present context a result in [3], which turns out to be related to Proposition 3.5.

Proposition 4.2. Suppose that Assumption 4.1 holds, and let $f$ be a function in $\mathcal{X}$. Then

(a) $\alpha^{n}\left(P^{n}-I\right) R_{\alpha} f=\left(\sum_{k=0}^{n-1} \alpha^{k}\right)(1-\alpha) R_{\alpha} f-\sum_{k=0}^{n-1} \alpha^{k} P^{k} f$ for all $\alpha \in$ $(0,1), n=1,2, \ldots$

(b) For every $n=1,2, \ldots$, the limit

$$
G_{n} f:=s-\lim _{\alpha \uparrow 1}\left(P^{n}-I\right) R_{\alpha} f
$$

exists if and only if $f$ is in $A(P)$, in which case [by (a) and (4.3)]

$$
G_{n} f=n \cdot \Pi f-S_{n} f \quad \forall n=1,2, \ldots,
$$

so that $s-\lim _{n \rightarrow \infty} G_{n} f / n=0$.

(c) For a given $f$ in $A(P)$, a function $h$ satisfies the P.E. (3.5) if and only if

$$
G_{n} f=P^{n} h-h \quad \forall n=1,2, \ldots
$$

Proof. (a) From (4.1), we get

$$
R_{\alpha}=I+\alpha P R_{\alpha} .
$$

Iteration yields

$$
R_{\alpha}=\sum_{k=0}^{n-1} \alpha^{k} P^{k}+\alpha^{n} P^{n} R_{\alpha} \quad \forall n=1,2, \ldots, 0<\alpha<1 .
$$


Subtracting $\alpha^{n} R_{\alpha}$ from both sides of this equation and recalling that

$$
1-\alpha^{n}=(1-\alpha) \sum_{k=0}^{n-1} \alpha^{k},
$$

we obtain (a).

(b) In (a) take both liminf and limsup as $\alpha \uparrow 1$ to get

$$
\begin{aligned}
s-\liminf _{\alpha \uparrow 1}\left(P^{n}-I\right) R_{\alpha} f & =n \cdot s-\liminf _{\alpha \uparrow 1}(1-\alpha) R_{\alpha} f-S_{n} f \\
& \leq n \cdot s-\limsup _{\alpha \uparrow 1}(1-\alpha) R_{\alpha} f-S_{n} f \\
& =s-\limsup _{\alpha \uparrow 1}\left(P^{n}-I\right) R_{\alpha} f .
\end{aligned}
$$

Thus, in view of (4.3), we conclude that (4.4)-(4.5) hold if and only if $f$ is in $A(P)$.

Finally, (c) follows from (4.5) and the equality in (3.7) [or (2.2) with $g=\Pi f]$.

With $n=1$, Proposition 4.2(a) implies the following expression for $(I-P) R_{\alpha}=R_{\alpha}(I-P)$ :

$$
\alpha R_{\alpha}(I-P) f=f-(1-\alpha) R_{\alpha} f \quad \forall f \in \mathcal{X} .
$$

Therefore, from (4.3) [or from (4.5) with $n=1$ ],

$$
s-\lim _{\alpha \uparrow 1} R_{\alpha}(I-P) f=(I-\Pi) f \quad \text { if } f \in A(P) .
$$

We will now employ (4.7) and (4.8) to obtain a result similar to Theorem 3.7 but using $R_{\alpha}$ instead of $A_{n}$.

First, following [2] (see also [33]) let $P_{0}$ denote the restriction of $P$ to $A(P)$ and define

$$
J f:=s-\lim _{\alpha \uparrow 1} R_{\alpha} f
$$

whenever the limit exists.

Theorem 4.3. Under Assumption 4.1:

(a) $\operatorname{Dom}(J)=\operatorname{Ran}\left(I-P_{0}\right)$ and $\operatorname{Ran}(J)=\operatorname{Ker}(\Pi)$.

(b) $f$ is in $\operatorname{Dom}(J)$ if and only if

$$
h=s-\lim _{\alpha \uparrow 1} R_{\alpha} f[=J f]
$$

is the unique solution of the Poisson equation $(g=0$ and $)$

$$
(I-P) h=f \quad \text { with } \Pi h=0 .
$$

(c) $f$ is in $\operatorname{Dom}(J)$ if and only if the pair $(g, h)$ with $g=\Pi f$ and $h$ as in (4.9) is the unique solution of the multichain P.E. (1.1) satisfying $\Pi h=0$. 
(d) The restriction of $J$ to $\operatorname{Ran}(J)=\operatorname{Ker}(\Pi)$ is the inverse of $I-P_{0}$, i.e.,

$$
(I-P) J f=f=J(I-P) f \quad \forall f \in \operatorname{Ker}(\Pi) .
$$

Proof. (a) To show that $\operatorname{Ran}\left(I-P_{0}\right) \subset \operatorname{Dom}(J)$, let $f$ be a function in $\operatorname{Ran}\left(I-P_{0}\right)$. Then there is a function $h$ in $A(P)$ such that $f=(I-P) h$ and (4.8) entails that $f$ is $\operatorname{Dom}(J)$ and

$$
J f=s-\lim R_{\alpha}(I-P) h=(I-\Pi) h .
$$

Suppose now that $f$ is in $\operatorname{Dom}(J)$ and let $J f=h$, i.e.,

$$
s \text { - } \lim _{\alpha \uparrow 1} R_{\alpha} f=h .
$$

Then multiplying the latter equality by $1-\alpha$ we obtain, by (4.3),

$$
\Pi f=s-\lim _{\alpha \uparrow 1}(1-\alpha) R_{\alpha} f=s-\lim _{\alpha \uparrow 1}(1-\alpha) \cdot h=0 .
$$

This in turn yields [by (4.8); recall also that $P$ is bounded]

$$
f=s-\lim _{\alpha \uparrow 1} R_{\alpha}(I-P) f=(I-P) \cdot J f=(I-P) h .
$$

Finally, by (4.13),

$$
h=s-\lim _{\alpha \uparrow 1} R_{\alpha} f=s-\lim _{\alpha \uparrow 1} R_{\alpha}(I-P) h \quad[\text { by }(4.14)],
$$

which, by (4.12), yields $h=h-\Pi h$; hence

$$
\Pi h=0 .
$$

Thus from (4.14) and (4.15) we conclude that $f$ is in $\operatorname{Ran}\left(I-P_{0}\right)$. This completes the proof of $\operatorname{Dom}(J)=\operatorname{Ran}\left(I-P_{0}\right)$.

On the other hand, note that (4.13) and (4.15) imply $\operatorname{Ran}(J) \subset \operatorname{Ker}(\Pi)$. Now let $h$ be in $\operatorname{Ker}(\Pi)$, and let $f:=(I-P) h$. Then (4.8) yields

$$
J f=s-\lim _{\alpha \uparrow 1} R_{\alpha}(I-P) h=(I-\Pi) h=h,
$$

i.e., $h$ is in $\operatorname{Ran}(J)$. In other words, $\operatorname{Ker}(\Pi) \subset \operatorname{Ran}(J)$.

(b) This follows from (a). Namely, the implication " $\Rightarrow$ " follows from (4.14) and (4.15), and the converse follows from (4.12). The uniqueness comes from Proposition 3.4.

(c) “ $\Rightarrow$ " If $f$ is in $\operatorname{Dom}(J)$, it follows from (4.5)-(4.6) and (2.2) that $(\Pi f, h)$ is an $f$-canonical pair. Thus, by Theorem 2.2 and Proposition 3.4, $(\Pi f, h)$ is the unique solution of (1.1) satisfying (3.6).

" $\Leftarrow$ " This follows from part (b): In (4.9) and (4.10), replace $f$ by $f-\Pi f$.

(d) The first equality in (4.11) follows from (4.9)-(4.10), and the second follows from (4.8) [or (4.12)]. 
REMARK 4.4. An informal way of arriving at the second equality in (4.3) as well as at

$$
J f=s-\lim _{\alpha \uparrow 1} R_{\alpha} f
$$

in (4.9) is as follows. In (4.1) replace $P^{k}$ by $\Pi+\left(P^{k}-\Pi\right)$ to get

$$
R_{\alpha}=\frac{\Pi}{1-\alpha}+\sum_{k=0}^{\infty} \alpha^{k}\left(P^{k}-\Pi\right) .
$$

This immediately suggests the second equality in (4.3) if $P^{k}-\Pi$ converges to zero sufficiently fast, for example, as in (3.9). In particular, using

$$
\alpha^{k}=1-(1-\alpha) \sum_{j=0}^{k-1} \alpha^{j} \quad \text { for } k=1,2, \ldots,
$$

by elementary calculations on (4.17) we get

$$
R_{\alpha}=\frac{\Pi}{1-\alpha}+H_{0}-(1-\alpha) \sum_{k=0}^{\infty} \alpha^{k} \sum_{n=k+1}^{\infty}\left(P^{n}-\Pi\right),
$$

where, as in Section 3,

$$
H_{0}:=\sum_{k=0}^{\infty}\left(P^{k}-\Pi\right)=s-\lim _{n \rightarrow \infty} \sum_{k=0}^{n-1}\left(P^{k}-\Pi\right) .
$$

Thus, if the sequence in (4.19) converges, (4.18) yields the second equality in (4.3) as well as (4.16) with $J f=H_{0} f$ for $f$ in $\operatorname{Ker}(\Pi)$. These calculations can be made precise even in the uniform (instead of the strong) operator topology; see, e.g., [20, 21, 24, 34]. Finally, note that expressions such as (4.18) can be used to obtain, for instance, rates of convergence of $(1-\alpha) R_{\alpha}$ to $\Pi$ as $\alpha \uparrow 1$.

5. Closing remarks and open problems. We have presented a detailed analysis of the problem of existence of solutions to the multichain P.E. (1.1) in a very general context, using the concept of canonical pairs (Section 2), Cesàro averages $A_{n}$ (Section 3), and $\alpha$-potentials $R_{\alpha}$ (Section 4). There remains, however, a lot to be done. In particular, some important open problems are the following.

1. To develop approximation schemes to solve (1.1), perhaps iteratively. If the state space $X$ is finite, some computational algorithms are availablesee the references in Remark 2.6. For the case of general $X$, some of the techniques in $[2,23,33,35]$ and their references might be useful.

2. Theorem 3.2 provides an "ergodic decomposition" of the domain $\mathcal{X}$ of $P$, expressing $\mathcal{X}$ as the (disjoint) union of $A(P)$ and its complement 
$\mathcal{X} \backslash A(P)$. This, in turn, is used to obtain the domain and range of the operators $\Pi, H, J$, and so on. It would be interesting to obtain a more precise form of these sets for particular classes of Banach spaces $\mathcal{X}$ (for instance, $\mathcal{X}$ an $L_{p}$ space) used in probability applications. There are, on the other hand, well known ergodic decompositions of the chain's state space $X$, such as, for instance, Hopf's and Doeblin's decompositions; see [8, 27, 37, 39]. These typically give more information on the Markov chain's probabilistic behaviour and, therefore, it would be important to relate them to the different sets appearing in Theorems 3.2, 3.7 and 4.3. In other words, the basic question is to find the relation (if any) between an ergodic decomposition of $\mathcal{X}$ and one of $X$.

3. An important issue in some Markovian control problems is to determine the validity of a Laurent expansion of the form [cf. (4.18)]

$$
R_{\alpha}=\Pi /(1-\alpha)+\sum_{n=0}^{\infty}(-\alpha)^{n} H^{n}
$$

where $H$ is the "deviation operator" in (3.10) [see also (3.13) and (2.15)]. To our knowledge, (5.1) has only been studied under very strong assumptions $[30,38,42]$, and it turns out to be related to a sequence of "nested" Poisson equations.

4. In Remark 3.3 we mentioned that, under appropriate hypotheses, the projection operator $\Pi$ is determined by a transition probability function $\Pi(x, B)$, which has nice implications. It would be useful to obtain a similar result for $(1-\alpha) R_{\alpha}$ [see (4.3)].

5 . In the unichain case, there is a well known relation between the existence of solutions to the Poisson equation and probabilistic conditions such as the Doeblin and Harris conditions [22, 31]. What can be said about this relation in the multichain case?

6. The results in this paper are for the "discrete-time" Poisson equation (1.1), in the strong topology. What are the corresponding results for the continuous-time case (when $P-I$ is replaced by the generator of a continuous-time Markov semigroup $P_{t}, t \geq 0$ ) and/or in the uniform or weak (operator) topologies? What can be said about the "adjoint" Poisson equation $\nu=\nu P^{*}, \mu\left(I-P^{*}\right)=\theta-\nu$, for a given "charge" $\theta$ in $\mathcal{X}^{*}$ ? (See $[2$, $21,23,24,29,33-36]$.)

\section{References}

[1] D. P. Bertsekas, Dynamic Programming: Deterministic and Stochastic Models, Prentice-Hall, Englewood Cliffs, NJ, 1987.

[2] P. L. Butzer and U. Westphal, The mean ergodic theorem and saturation, Indiana Univ. Math. J. 20 (1971), 1163-1174. 
[3] R. Cavazos-Cadena, A note on the vanishing interest rate approach in average Markov decision chains with continuous and bounded costs, Systems Control Lett. 24 (1995), 373-383.

[4] K. S. Chan, A review of some limit theorems of Markov chains and their applications, in: H. Tong (ed.), Dimension, Estimation and Models, World Scientific, Singapore, 1993, 108-135.

[5] N. Dunford and J. T. Schwartz, Linear Operators, Part I, Wiley-Interscience, New York, 1957.

[6] E. B. Dynkin and A. A. Yushkevich, Controlled Markov Processes, Springer, New York, 1979.

[7] R. Emilion, Mean-bounded operators and mean ergodic theorems, J. Funct. Anal. 61 (1985), 1-14.

[8] S. R. Foguel, The Ergodic Theory of Markov Processes, Van Nostrand Reinhold, New York, 1969.

[9] A. G. Gibson, A discrete Hille-Yosida-Phillips theorem, J. Math. Anal. Appl. 39 (1972), 761-770.

[10] P. Glynn and S. P. Meyn, A Lyapunov bound for solutions of Poisson's equation, Ann. Probab. 24 (1996), 916-931.

[11] O. Hernández-Lerma, Adaptive Markov Control Processes, Springer, New York, 1989.

[12] O. Hernández-Lerma and J. González-Hernández, Infinite linear programming and multichain Markov control processes in uncountable spaces, SIAM J. Control Optim. 36 (1998), 313-335.

[13] O. Hernández-Lerma and J. B. Lasserre, Discrete-Time Markov Control Processes: Basic Optimality Criteria, Springer, New York, 1996.

[14] - , Policy iteration for average cost Markov control processes on Borel spaces, Acta Appl. Math. 47 (1997), 127-154.

[15] - Ergodic theorems and ergodic decomposition of Markov chains, ibid. 54 (1998), 99-119.

[16] - Existence of solutions to the Poisson equation in $L_{p}$ spaces, in: Proc. 35th IEEE-CDC, Kobe, Japan, 1996, vol. 4, 4190-4195.

[17] O. Hernández-Lerma, R. Montes-de-Oca and R. Cavazos-Cadena, Recurrence conditions for Markov decision processes with Borel state space: a survey, Ann. Oper. Res. 28 (1991), 29-46.

[18] A. Hordijk and F. Spieksma, A new formula for the deviation matrix, in: F. P. Kelly (ed.), Probability, Statistics and Optimization, Wiley, New York, 1994, 497-507.

[19] M. Iosifescu, A basic tool in mathematical chaos theory: Doeblin and Fortet's ergodic theorem and Ionescu Tulcea and Marinescu's generalization, in: Contemp. Math. 149, Amer. Math. Soc., 1993, 111-124.

[20] S. Karlin, Positive operators, J. Math. Mech. 8 (1959), 907-937.

[21] M. Lin, On the uniform ergodic theorem, II, Proc. Amer. Math. Soc. 46 (1974), $217-225$.

[22] —, Quasi-compactness and uniform ergodicity of Markov operators, Ann. Inst. H. Poincaré Sect. B 11 (1975), 345-354.

[23] M. Lin and R. Sine, Ergodic theory and the functional equation $(I-T) x=y$, J. Operator Theory 10 (1983), 153-166.

[24] Yu. Lyubich and J. Zemánek, Precompactness in the uniform ergodic theory, Studia Math. 112 (1994), 89-97.

[25] A. M. Makowski and A. Shwartz, On the Poisson equation for countable Markov chains, Tech. Rept., Dept. of Electrical Engineering, Univ. of Maryland, College Park, MD, 1994. 
[26] M. Métivier et P. Priouret, Théorèmes de convergence presque sure pour une classe d'algorithmes stochastiques à pas décroissant, Probab. Theory Related Fields 74 (1987), 403-428.

[27] S. P. Meyn and R. L. Tweedie, The Doeblin decomposition, in: Contemp. Math. 149, Amer. Math. Soc., 1993, 211-225.

[28] —, Markov Chains and Stochastic Stability, Springer, London, 1993.

[29] E. Nummelin, On the Poisson equation in the potential theory of a single kernel, Math. Scand. 68 (1991), 59-82.

[30] M. L. Puterman, Markov Decision Processes, Wiley, New York, 1994.

[31] D. Revuz, Markov Chains, North-Holland, Amsterdam, 1984.

[32] S.-Y. Shaw, Ergodic projections on continuous and discrete semigroups, Proc. Amer. Math. Soc. 78 (1980), 69-76.

[33] - Mean ergodic theorems and linear functional equations, J. Funct. Anal. 87 (1989), $428-441$.

[34] - Uniform convergence of ergodic limits and approximate solutions, Proc. Amer. Math. Soc. 114 (1992), 405-411.

[35] - Convergence rates of ergodic limits and approximate solutions, J. Approx. Theory 75 (1993), 157-166.

[36] R. Syski, Ergodic potential, Stochastic Process. Appl. 7 (1978), 311-336.

[37] W. Szczechla, On ergodic averages and absorbing sets for positive contractions in $L_{1}$, J. Math. Anal. Appl. 194 (1995), 560-568.

[38] H. M. Taylor, A Laurent series for the resolvent of a strongly continuous stochastic semigroup, Math. Programming Stud. 6 (1976), 258-263.

[39] K. Yosida, Functional Analysis, 6th ed., Springer, Berlin, 1980.

[40] K. Yosida and S. Kakutani, Operator-theoretical treatment of Markoff processes and mean ergodic theorem, Ann. of Math. 42 (1941), 188-228.

[41] A. A. Yushkevich, On a class of strategies in general Markov decision models, Theory Probab. Appl. 18 (1973), 777-779.

[42] - Blackwell optimal policies in a Markov decision process with a Borel state space, Z. Oper. Res. 40 (1994), 253-288.

Departamento de Matemáticas

CINVESTAV-IPN

A. Postal 14-740

México, D.F. 07000, México

E-mail: ohernand@math.cinvestav.mx

\section{LAAS-CNRS}

7 Av. du Colonel Roche

31077 Toulouse Cedex 4, France

E-mail: lasserre@laas.fr

Received on 6.11.2000;

revised version on 26.2.2001 